\title{
Integrating HSE Quality Systems Using a Hazards Priority Report Based on the AHP Methodology
}

\author{
L. Compagno ${ }^{1}$, D. D’Urso ${ }^{1}$, A. Latora ${ }^{1}$, B. Martino ${ }^{2}$, F. Nicolosi ${ }^{2}$, G. Aprile ${ }^{2}$ \\ ${ }^{1}$ Università degli Studi di Catania, Italy \\ ${ }^{2}$ Isab srl, Syracuse, Italy
}

\begin{abstract}
The protection of the health and safety of workers and the respect for the environment play a key role in the management of the industrial plants.

The health, safety and environment management systems offer a methodological contribution that leads to a continuous-loop process re-engineering and drive improvements based on different criteria.

Thus, a multi-criteria evaluation of environmental and safety performances of industrial plants is required and allows a real integration of sustained efforts.

In this scenario the hierarchical analysis (AHP) can allow an efficient synthesis of the environmental impacts and risks which are attributable to each production unit.

Along this dimension of research, a hierarchical structure, related to the context of an oil refinery plant, has been codified.

More in particular, the information content of the risk assessment and of the environmental analysis processes fed the hierarchical composition process of Saaty obtaining the assignment of a holistic judgment to each production unit that is responsible.

This judgment determines the priority of allocation of maintenance resources and overcomes the limitations of risk matrices that are so focused on single hazard or environmental aspect to be short-sighted.

The method also enables to engage the corporate management and institutional stakeholders involved in the assessment of the weights to be assigned to each criterion of risk and environmental assessment through the adoption of the group decision making.

Keywords: group decision making, hazard matrix, HSE quality systems integration.
\end{abstract}

\section{Introduction}

The Quality Management Systems offer a significant contribution to the proper management of industrial processes, their environmental impacts, risks to the health and safety of the operators.

The contribution of Quality Systems has become more significant since the publication of the ISO 9001:2000 standard, which introduced the concept of continuous loop reengineering.

In fact, since the publication of the above-mentioned standard, Quality systems are proposed as real management tools whose effectiveness, in the context of operations, is placed against the interpretation that many bureaucratic and formal corporate players had been given.

Precisely, Quality systems base their structure on the fundamental paradigm that animates the procedure of reorganization of processes such as: the analysis of the state of the art, the definition and subsequent measurement of appropriate performance indicators, the diagnosis of the state of the art, in particular by comparison with a predetermined 
benchmark or with a predetermined target level, and finally, the cure or the definition of an improvement plan.

The continuous loop process analysis, thus introduced, is extended to all the relevant dimensions of production systems such as technological, organizational, managerial and human resources.

The Quality Systems help the operational management of key business processes, including production management, environmental management and management of health and safety of the operators.

The simultaneous presence of these instruments has led to their integration in an attempt to improve the efficiency and ultimately to operate the main activities of analysis and management in a holistic manner so as to obtain both a complete vision of the company and economies of scale.

Along this path of evolution, the process analysis, which is common to all quality systems, has become the real lever of integration that can significantly reduce the effort required for the study of the processes and the prioritization of interventions for improvement.

The latter option is particularly critical: the outcome of the analysis of the process, both for environmental analysis or related to the risk assessment for operators, is to define an index that measures the risk associated with an environmental aspect or with a danger; in general, this indicator is obtained by means of the product of the probability of occurrence and the impact of environmental or risk in question.

The limit of the above mentioned process is the lack of integration; usually rankings of environmental impacts or hazards for the workers are known without an instrument which, instead, enables assigning to each production unit a holistic judgement that represents the combined results of all analyzes conducted.

This lack of integration of the analysis of quality systems results in ineffective improvement planning.

The present study applies the method of hierarchical composition proposed by Saaty (AHP) to formulate the desired holistic judgment on the maintenance priority by which resources are assigned to each production unit of an oil refinery.

In the following, after a brief review of literature, the case study is presented.

The results of the assessments of the environmental impacts and risks for the health and safety of operators feed a process of hierarchical composition which gives a priority for maintenance or improvement (priority reports) to each production unit. Within the organizational structure of the company, the hierarchical structure has been introduced in order to implement the priority report based on a group decision making process among corporate and institutional stakeholders.

\section{Literature Review and state of the art}

The implementation of management systems, such as those of Quality, Environment and Health and Safety for operators, commits resources to update the analysis of the state of art of the systems, for the development of the same and for training.

The usefulness of their adoption is now widely agreed in the industrial field [1]. The Quality systems have many common aspects resulting from the same methodological framework defined by the Deming cycle [2], [3], [6], [8]. 
Then it is natural that their joint application raises the question of how to avoid duplication, take advantage of the potential synergies, and ensure the communion of the objectives.

Environment, Health and Safety systems intervene, in particular, on the same processes. So their integration is now a must which is reached, in most cases, by a process of integration of the two systems once already implemented or, more rarely, by an initial integrated design [4], [5].

The environmental and safety culture must therefore be used within a process of integration which is not the addition of a series of documents; integration, in fact, is not a standard but it is a process that management develops according to its own criteria in order to reach the overall efficiency [7].

The ISO 14001:2004 standard defines the requirements that an environmental management system must have based on five pillars:

- Environmental policy; it reflects the organization's commitment to environmental management and continuous improvement;

- Planning; starting from the analysis of the existing, it leads to the formulation of objectives, targets and program development;

- Implementation and operation of the system; to this purpose the organization must develop its capacity and make available the necessary resources;

- Verification (of the operations, performance, requirements, compliance, management system) and the implementation of corrective actions;

- Management review; it's aimed to review and continually improve its environmental management system.

The BS OHSAS 18001:2007 standard establishes, on the other hand, the requirements that a system for managing health and safety of workers must have. However, it is mandatory according to Italian Legislative Decree 334/99 which transposes the European Directive n. 82 of 1996 for process plants subjected to risk of a major accident.

The above mentioned standards have the same structure and this certainly suggests and facilitates their integrated application.

The integration of environmental, health and safety management systems is a complex activity that takes place according to three methods that are not mutually exclusive: organizational integration, topics integration and processes integration.

The process integration is certainly the more general and thorough; it leads to join the environmental and risk analysis at least for the following reasons:

- they lead to the diagnosis of the state of the processes as the antecedent of any further improvement intervention;

- they allow, in turns, to highlight further opportunities for integration.

The opportunities for integration between the two analyzes are manifold.

First of all the formalities required by the BS OHSAS 18001:2007 standard correspond to those required by binding legal regulation (Italian Legislative Decree no. 81/2008). Furthermore, the analysis of the work environment in terms of emergencies that affect the systems safety and the environmental coincides for the two systems; materials and hazardous waste are also simultaneously sources of hazards and environmental aspects.

The Italian Legislative Decree no. 81/2008 introduced the requirements to conduct the assessment of the risks associated with industrial activity and the reduction of the risks identified through appropriate preventive and protective measures. 
The risk and environmental issues assessment thus represents a fundamental tool to learn about activities, materials, equipments which may harm according to the methods of development, or to the characteristics of the work environment. The process of risk assessment consists normally of three stages.

The starting point is to identify potential sources of risk of the activity. This develops through the detailed description of the work cycle and operating activities; this allows for the complete mapping of the operations and processes carried out in the working environment considered and to identify possible sources of risk to the health and safety of personnel.

Secondly, it is necessary to determine whether, in carrying out a specific activity, the sources of potential risk, as identified in the previous phase, involve a real risk to the operator, taking into account the characteristics of the work (operating modes, the characteristics of exposure, existing protection measures, etc.).

Finally, the estimate of the risk arising from the work is performed.

Risk reduction can be achieved by decreasing the probability of occurrence of a certain expected damage (prevention) and/or mitigating the consequences (protection). The intervention of risk reduction is subject to the definition of an acceptable level of risk Ra, whereby prior shall be deemed, for the purposes of the action, all the situations that present a risk level $\mathrm{R}>\mathrm{Ra}$.

\section{Hypotheses/Objectives}

The process described so far allows defining a priority both for action and for resources allocation. The limit belongs to the analysis that is focused on the mitigation of each environmental aspect or each risk for operators without taking into account a higher point of view that assigns to each production unit a holistic judgment of risk, for operators and environment, in order to more effectively drive the improvement process.

The improvement we want to reach implies the definition of a risk measure for the entire production unit that is the synthesis of the entire information content of risk analysis and environmental analyses.

We propose, therefore, a hierarchical methodology that finalizes the process of risk and environmental aspects assessment.

A hierarchical structure based on the AHP may allow a more flexible evaluation of each criterion of analysis and to this end the hierarchical structure lends itself to be at the core of a decision-making group; this latter can finalize the correct set of weights of the evaluation criteria.

In the following the results of risk analysis and environmental analysis will take as input data.

It is also hypothesized that risk and environmental analyses count independent risks and environmental issues so that it may be sufficient to use the method of Analytic Hierarchy Process.

\section{Research Design/Methodology}

The preparation of the decision-making model and the hierarchical structure is dedicated to the case study of an oil refinery. Here, according to the procedure of the management system of the Health and Safety of the operators, the risk assessment is conducted.

The analysis follows the following steps: definition of the working group, identification and collection of information, drawing of the Risk Assessment report, dissemination of 
the report to all the functions involved, plan update of information and training of workers.

The process of gathering information and preparing the document for the risk assessment involves:

- the partition of the system in homogeneous areas (production units) in terms of danger; henceforth these areas are identified and numbered by the index k;

- the census of the dangers of each area; six classes of hazards are considered: ordinary, special, ergonomic, process-related, organizational and work-related stress; the code $\mathrm{HC}_{\mathrm{i}, \mathrm{k}}$ identifies the type of danger, $\mathrm{i}$, for each homogeneous area of the plant $\mathrm{k}$;

- the census of operators exposed and the analysis of tasks;

- the risk assessment; this evaluation aims to determine a priority index for any followup action for improvement or mitigation; since the type of hazards, the exposure mode, the nature and intensity of the damage form a non-homogeneous set, we prefer to identify an evaluation criteria dedicated to each type of danger. For the identification of the priorities of the measures of prevention and protection to be adopted, a scale of three levels of intensity was defined according to the following semantics: acceptable, tolerable, improvable;

- the definition of additional safeguards;

- the preparation of the program of improvement.

The appendix lists the identified hazards (tab.a1; $\mathrm{HSC}_{\mathrm{i}, \mathrm{k}}$ ); figure 1 shows the results of the risk assessment for a part of the homogeneous areas and facilities identified (the colours correspond to the accepted thresholds of risk : acceptable $=$ white, tolerable $=$ light gray, dark gray $=$ to be improved).

According to a further procedure, the environmental aspects related to the activities carried out within the plant are identified in order to determine which of them have or can have significant impacts on the environment and in order to identify appropriate systems of control and prevention [9], [10]. The analysis follows the following steps:

- identifying, for each environmental issue, the balance of the flows of matter and energy;

- qualitative and quantitative identification of environmental aspects associated with each flow;

- assessment of the significance of each environmental aspect by means of a quantitative evaluation based on environmental, social, technical and economic criteria.

The analysis takes into account the different operating conditions of the system: normal, abnormal and emergency conditions.

Environmental issues considered $\left(\mathrm{EC}_{\mathrm{i}, \mathrm{k}}\right)$ and related to each production unit are: contamination, consumption of natural resources, emissions in the atmosphere, greenhouse gas emissions, energy consumption, visual impact, odours, waste, noise and water discharges.

The evaluation phase of the environmental issues, in order to identify the significant ones, is conducted according to a scoring method based on the following criteria of environmental, social and technical-economic $\left(\mathrm{SEC}_{\mathrm{i}, \mathrm{k}}\right)$ :

the hazard $(\mathrm{H})$ or potential severity of the impact on the environment, the contribution (C) relative to the overall system, the report of stakeholders (S) or the sensitivity of the parties concerned specific aspect, the vulnerability environmental (V) which is the vulnerability of the ecosystem specific impact, the frequency $(F)$ with which the impact 
object may occur, the improvement potential (I) which represents the degree of improvement.

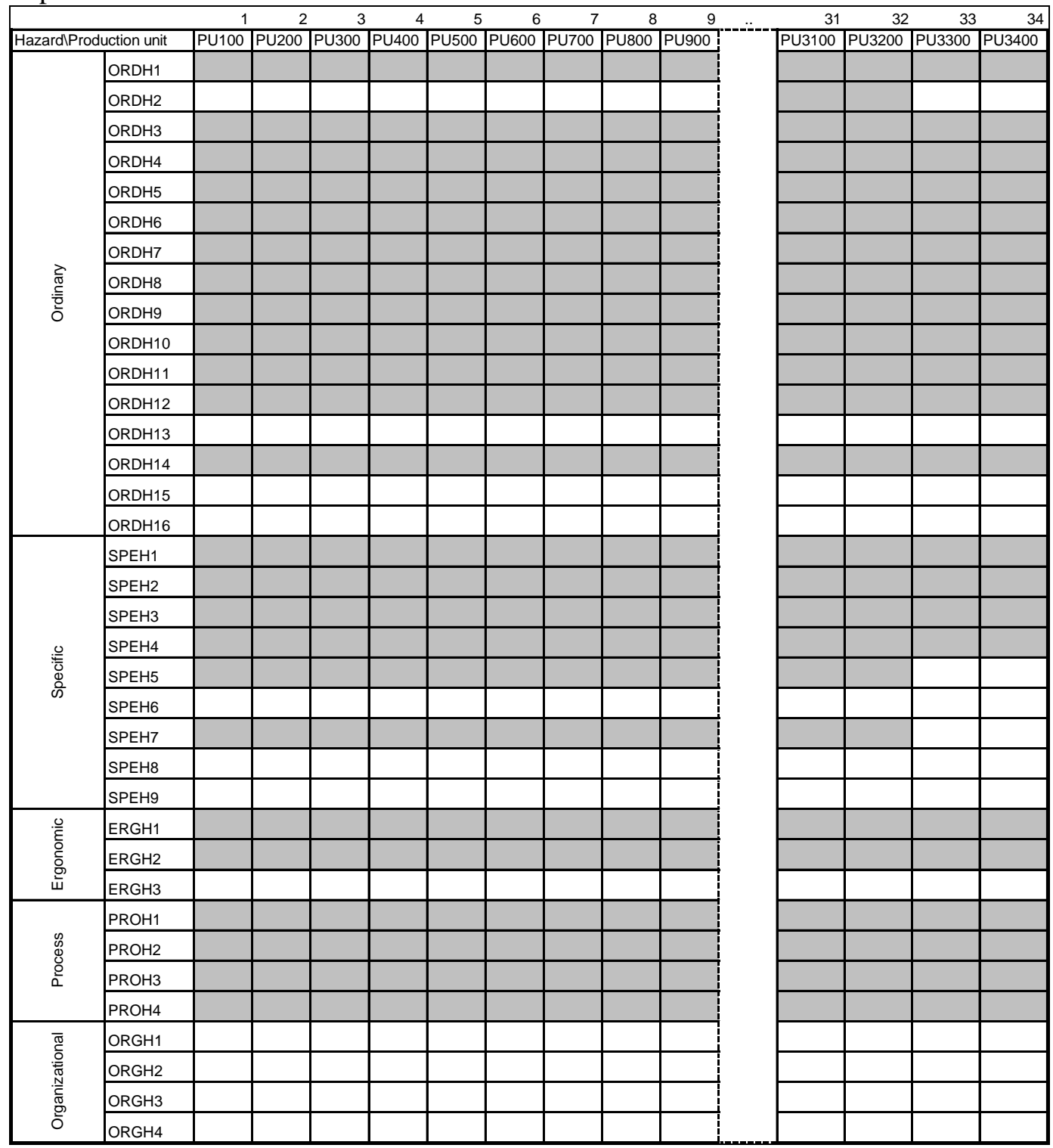

Fig.1 Risk Assessment for each homogeneous area identified (the risk matrix has 37 hazards for each of the 34 production unit, and then 1258 are potential measures for improvement).

For each environmental aspect a numeric index for each evaluation criterion has been assigned by using a four values semantic scale, [1..4].

The indices have been identified on the basis of both quantitative and qualitative criteria. They were then used for the construction of two indices of evaluation in order to classify the environmental importance and to assign priority to the actions on the individual assets in the formulation of environmental programs for the site.

In particular, the importance of the environment issue is determined by the formula: Importance of environmental issue $=\mathrm{IEI}=[\mathrm{H}+\mathrm{C}+\mathrm{S}] \times \mathrm{V} \times \mathrm{F}$

The priority of action on every aspect is indicated by the index found by the formula: International Symposium of the Analytic Hierarchy 
Priority of Intervention $=\mathrm{PI}=\mathrm{IEI} \times \mathrm{I}$

Figure 2 shows the results of the environmental review; this has 165 environmental aspects of which 25 are considered significant; each aspect refers to one or more plants.

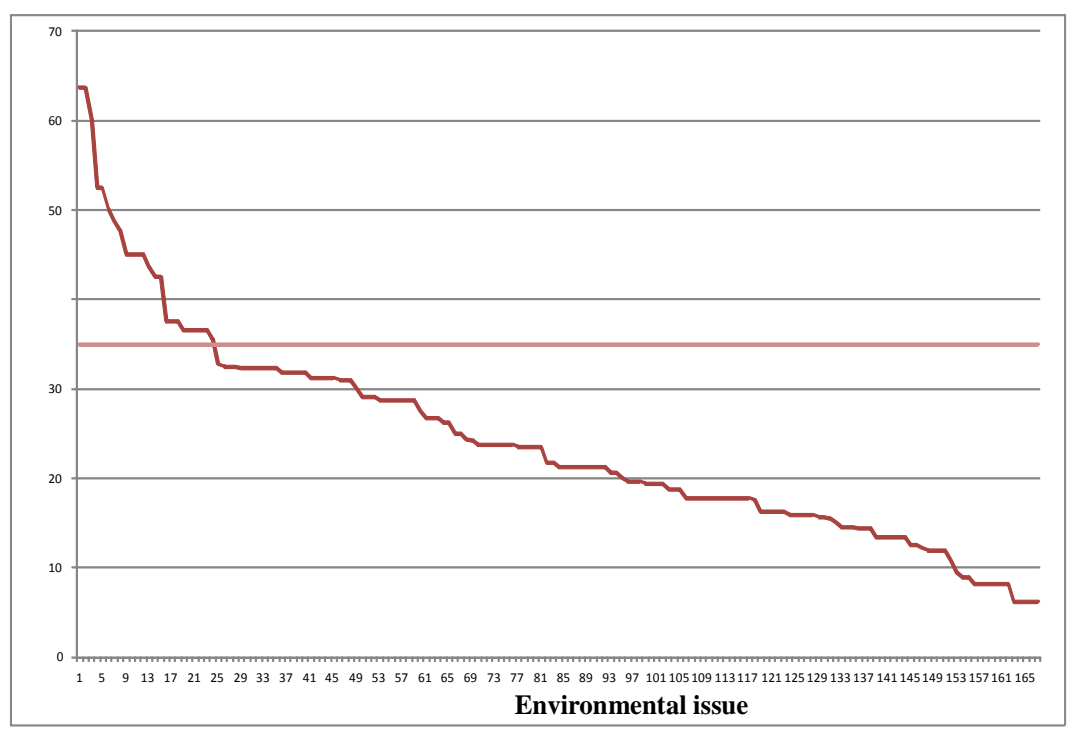

Fig.2 Priorities of action for each environmental issue; the limit of significance is emphasized.

\section{Data/Model Analysis}

Based on the two previous analyses, two hierarchical structures, one for the risk assessment and the other for the evaluation of environmental aspects, have been designed and are represented in Figure 3 and 4.

The Figures 3 and 4 allow to verify that the process of allocation of risks in each business unit is via a three-level hierarchical composition.

If the goal is the allocation of a risk priority to each plant, the criteria under which the risk analysis and environmental analysis are conducted $\left(\mathrm{HC}_{\mathrm{k}}\right.$ or $\left.\mathrm{EC}_{\mathrm{k}}\right)$ belong to the first level; the sub-criteria or the elements involved in the risk assessment $\left(\mathrm{HSC}_{\mathrm{k}}\right.$ or $\left.\mathrm{ESC}_{\mathrm{k}}\right)$ belong to the second level; at the third level the alternatives are placed that are the subject of prioritization.

The method of hierarchical composition, having established local weights of each criterion or sub-criterion of evaluation on the basis of a process of pair wise comparison, allows assigning the desired holistic judgment $\left(\mathrm{PI}_{\mathrm{i}}\right)$ to each plant according to the $\mathrm{i}$-th matrix relation:

$\mathrm{PI}_{\mathrm{i}}=\left[\mathrm{WHC}_{\mathrm{k}}\right] \cdot\left[\mathrm{WHSC}_{\mathrm{k}, \mathrm{j}} \mathrm{HSC}_{\mathrm{j}}\right]$

The weights of each evaluation criterion $\mathrm{WHC}_{\mathrm{k}}$ were determined by the method of pair wise comparisons, and in particular by operating the average of the opinions expressed by each of the members of the working group responsible for the process of risk assessment and environmental aspects (see table 2). The weights of the local sub-criteria, $\mathrm{WHSC}_{\mathrm{k}}$, were also evaluated by using the pair wise comparison procedure.

Finally the values of risk associated with each hazard were assumed from the analysis of risk that is evaluated in the risk assessment report; the local weight of each hazard is equal to the ratio between the risk value and the sum of all risks that belongs to the same sub-criterion. 
Tab.2 Weights of hazard criteria, $\mathrm{WHC}_{\mathrm{k}}$, assessment (C.I.=0,082)

\begin{tabular}{|l|c|c|c|c|c|c|}
\hline \multicolumn{1}{|c|}{ Hazard } & Ordinary & Ergonomics & Specific & Process & Organizational & WHC $_{\mathbf{k}}$ \\
\hline Ordinary & 1 & $1 / 3$ & $1 / 7$ & $1 / 9$ & $1 / 5$ & $3 \%$ \\
\hline Ergonomic & 3 & 1 & $1 / 7$ & $1 / 9$ & $1 / 5$ & $5 \%$ \\
\hline Specific & 7 & 7 & 1 & $1 / 3$ & 3 & $27 \%$ \\
\hline Process related & 9 & 9 & 3 & 1 & 5 & $51 \%$ \\
\hline Organizational & 5 & 5 & $1 / 3$ & $1 / 5$ & 1 & $14 \%$ \\
\hline
\end{tabular}

Finally the values of risk associated with each hazard were assumed from the analysis of risk that is evaluated in the risk assessment report; the local weight of each hazard is equal to the ratio between the risk value and the sum of all risks that belongs to the same sub-criterion.

Figure 5 shows the outcome of the hierarchical composition that corresponds to the hierarchical priority reports obtained by the equation (3).

It should be noted that the value of holistic merit, taking into account the environmental aspects and those related to risk, is the arithmetic average of the value found for the two hierarchical compositions presented since it is believed that the environmental assessment and the safety have the same weight.

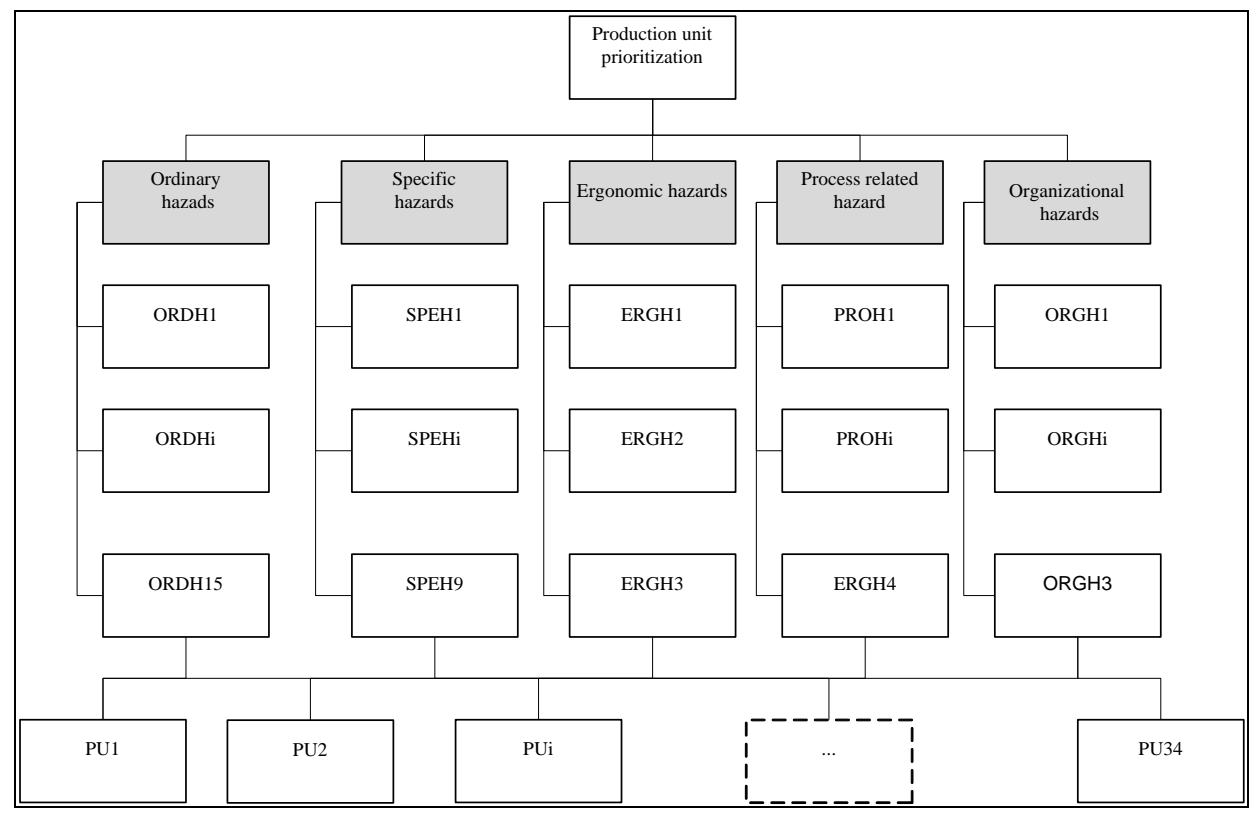

Fig.3 Analytic hierarchy structure designed in order to assign risks to each production unit. 


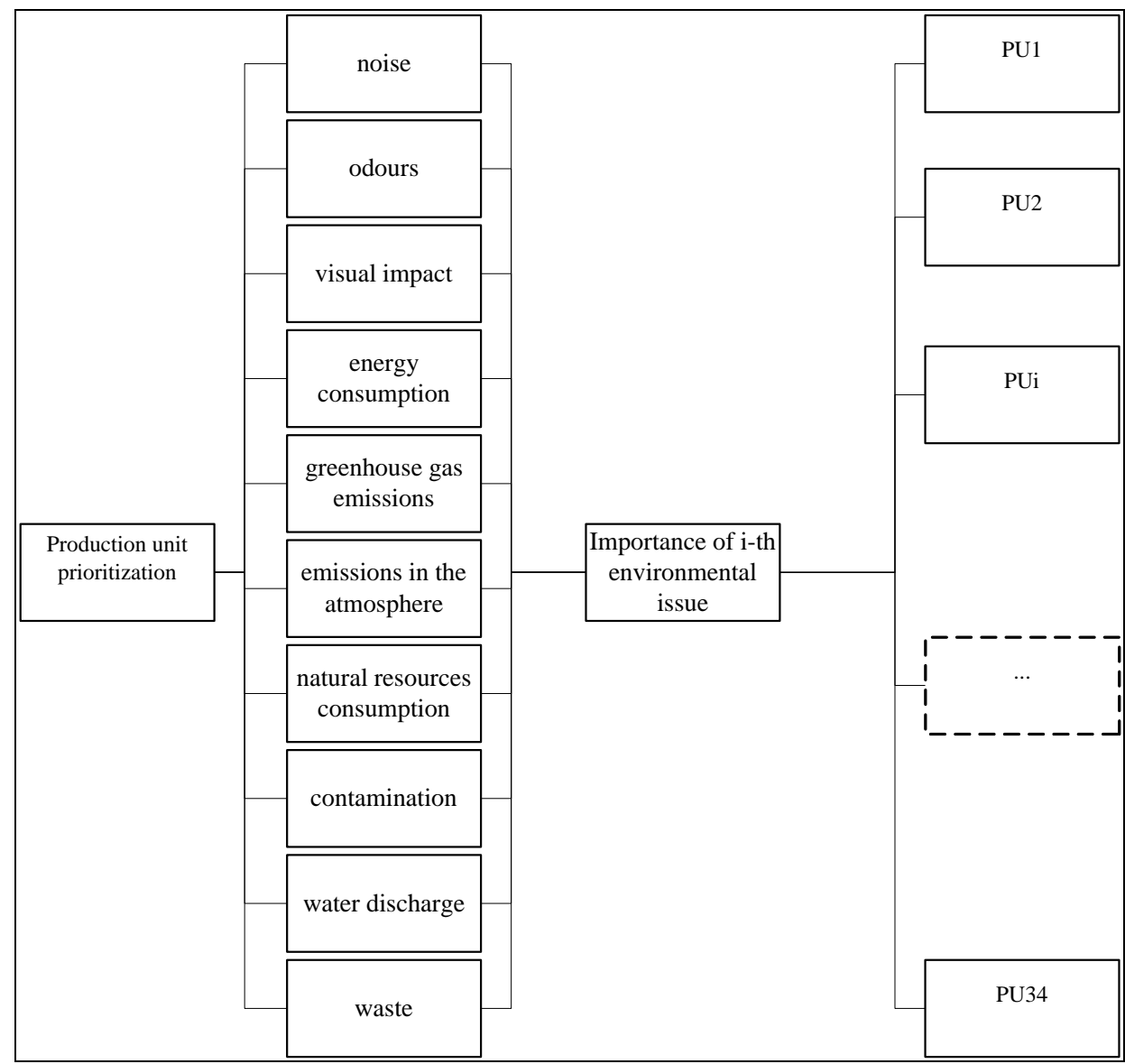

Fig.4 Analytic hierarchy structure designed in order to assign environmental risks to each production unit.

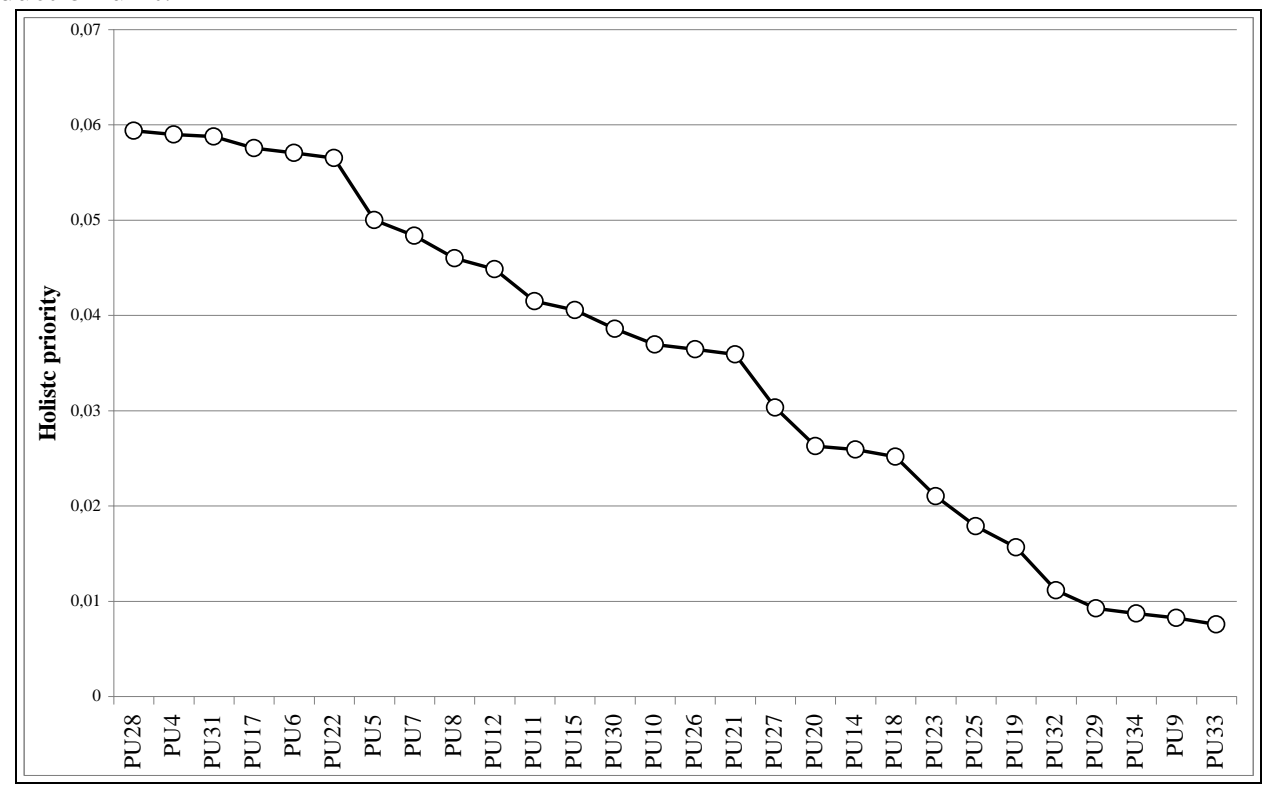

Fig.5 Holistic priority for each production unit. 


\section{Limitations}

The study gives a holistic assessment of risk to each production unit; it is not yet proven that the allocation of resources according to an index of priority dedicated to the single production unit is more efficient and effective than the allocation of resources on a set of improvement interventions transverse to each plant. A comparison between the current scenario and the one proposed would allow verifying the performance of the proposed solution.

\section{Conclusions}

The environmental, health and safety risk assessment of oil refinery plants, which are subjected to the risk of a major accident, is both valuable and critical.

This assessment process conducted on the field adheres to the binding legal framework and explores each aspect using analytical and focused methods.

The state of the art finalization of the analysis allows the prioritization of a set of interventions to improve every single aspect.

This point of view seems to be too focused and does not take into account all the arguments leading to a general assessment of the risk, to the health and safety of workers and the environment, of each production unit.

The use of a hierarchical-analytics has enabled to integrate the deep cultural content of the risk assessment in order to obtain a synthesis useful to a more effective intervention planning for improvement.

The hierarchical structure helps the decision making process and may enable involving all decision makers among company management and institutional stakeholders.

\section{Key References}

1. H.J. Pasman, B. Knegtering, W.J. Rogers, A holistic approach to control process safety risks: Possible ways forward, Reliability Engineering \& System Safety, Volume 117, September 2013, Pages 21-29, ISSN 0951-8320.

2. A. N. Haddad, C.V. Morgado, D. I. DeSouza, Health, Safety and Environmental Management Risk Evaluation Strategy: Hazard Matrix Application Case Studies; Industrial Engineering and Engineering Management, 2008. IEEM 2008. IEEE International Conference on Digital Object Identifier: Publication Year: 2008, Page(s): 1314 - 1318.

3. Alena Labodová, Implementing integrated management systems using a risk analysis based approach, Journal of Cleaner Production, Volume 12, Issue 6, August 2004, Pages 571-580, ISSN 0959-6526.

4. Faisal I Khan, Mahmoud M Haddara, Risk-based maintenance (RBM): a quantitative approach for maintenance/inspection scheduling and planning, Journal of Loss Prevention in the Process Industries, Volume 16, Issue 6, November 2003, Pages 561-573, ISSN 0950-4230.

5. Hans J Pasman, Risk informed resource allocation policy: safety can save costs, Journal of Hazardous Materials, Volume 71, Issues 1-3, 7 January 2000, Pages 375-394, ISSN 0304-3894. 
6. J. Tixier, G. Dusserre, O. Salvi, D. Gaston, Review of 62 risk analysis methodologies of industrial plants, Journal of Loss Prevention in the Process Industries, Volume 15, Issue 4, July 2002, Pages 291-303, ISSN 0950-4230.

7. A. Azadeh, I.M. Fam, M. Khoshnoud, M. Nikafrouz, Design and implementation of a fuzzy expert system for performance assessment of an integrated health, safety, environment (HSE) and ergonomics system: The case of a gas refinery, Information Sciences, Volume 178, Issue 22, 15 November 2008, Pages 42804300, ISSN 0020-0255.

8. Nijs Jan Duijm, Cécile Fiévez, Marko Gerbec, Ulrich Hauptmanns, Myrto Konstandinidou, Management of health, safety and environment in process industry, Safety Science, Volume 46, Issue 6, July 2008, Pages 908-920, ISSN 0925-7535.

9. Adel Badri, Sylvie Nadeau, André Gbodossou, Proposal of a risk-factor-based analytical approach for integrating occupational health and safety into project risk evaluation, Accident Analysis \& Prevention, Volume 48, September 2012, Pages 223-234, ISSN 0001-4575.

10. Aliakbar Golshani Asl, and Farzaneh Ahmadzadeh, Risk Prioritization Based on Health, Safety and Environmental Factors by Using Fuzzy FMEA, International Journal of Mining, Metallurgy \& Mechanical Engineering (IJMMME), Volume 1, Issue 4 (2013), ISSN 2320-4052; EISSN 2320-4060. 


\section{Appendices}

List of considered hazards.

\begin{tabular}{|c|c|}
\hline & Ordinary hazards \\
\hline ORDH1 & Cutting tools \\
\hline$\overline{\text { ORDH2 }}$ & Machinery \\
\hline ORDH3 & Fixed and mobile obstacles \\
\hline ORDH4 & Falls from height and falls deeply \\
\hline ORDH5 & Uneven pavement and/or slippery (slipping, loss of balance, falling over, tripping) \\
\hline ORDH6 & Equipment/tools: screening of splinters and fragments (including water jets) \\
\hline ORDH7 & Objects/materials altitude (weights drop) \\
\hline ORDH8 & Weather conditions and microclimate \\
\hline ORDH9 & Lighting (low/high) \\
\hline ORDH10 & Warm and cold surfaces and/or materials (including jets of liquids or vapors) \\
\hline ORDH11 & Closed spaces \\
\hline ORDH12 & Insect bites \\
\hline ORDH13 & Road accident \\
\hline ORDH14 & Fall in sea \\
\hline \multirow[t]{2}{*}{ ORDH15 } & Static electricity accumulation \\
\hline & Ergonomic hazards \\
\hline ERGH1 & Manual handling of loads \\
\hline ERGH2 & Incorrect operational postures \\
\hline \multirow[t]{2}{*}{ ERGH3 } & VDT \\
\hline & Specific hazards \\
\hline SPEH1 & Chemicals hazardous to health \\
\hline SPEH2 & Hazardous chemical agents for safety \\
\hline SPEH3 & Carcinogens / mutagens \\
\hline SPEH4 & Noise, Ultrasound, Infrasound \\
\hline SPEH5 & Vibrations \\
\hline SPEH6 & Ionizing radiation \\
\hline SPEH7 & Non-ionizing radiation \\
\hline SPEH8 & Inert powders \\
\hline \multirow[t]{2}{*}{ SPEH9 } & Biological agents \\
\hline & Process related hazards \\
\hline PROH1 & Fire \\
\hline PROH2 & Explosion \\
\hline PROH3 & Uncontrolled release of toxic substances \\
\hline \multirow[t]{2}{*}{ PROH4 } & Uncontrolled release of thermal energy or mechanical \\
\hline & Organizational hazards \\
\hline ORGH1 & Lack in the definition of roles and responsibilities \\
\hline ORGH2 & Lack in information activities, education and training \\
\hline ORGH3 & Lack in the activity of audits and / or inspections \\
\hline
\end{tabular}

